\title{
Towards answering the optimal palliative fractionation conundrum: single- versus multi-fraction radiation therapy for spinal cord compression
}

\author{
James E. Han ${ }^{1}$, Robert H. Press ${ }^{2}$, Shaakir Hasan ${ }^{2}$, J. Isabelle Choi ${ }^{2}$, Charles B. Simone II $^{2}$ \\ ${ }^{1}$ Department of Radiation Oncology, University of Arizona, Tucson, AZ, USA; ${ }^{2}$ Department of Radiation Oncology, New York Proton Center, New \\ York, NY, USA \\ Correspondence to: Charles B. Simone II, MD. New York Proton Center, 225 East 126th Street, New York, NY 10035, USA. \\ Email: csimone@nyproton.com. \\ Provenance and Peer Review: This article was commissioned by the editorial office, Annals of Palliative Medicine. The article did not undergo external \\ peer review. \\ Comment on: Hoskin PJ, Hopkins K, Misra V, et al. Effect of Single-Fraction vs. Multifraction Radiotherapy on Ambulatory Status Among Patients \\ With Spinal Canal Compression From Metastatic Cancer: The SCORAD Randomized Clinical Trial. JAMA 2019;322:2084-94.
}

Submitted Apr 20, 2020. Accepted for publication May 08, 2020.

doi: 10.21037/apm-20-990

View this article at: http://dx.doi.org/10.21037/apm-20-990

Patients with metastatic epidural spinal cord compression (MESCC) often present with loss of neurologic function, pain, and reduction in quality of life, all of which can be improved with palliative radiation therapy (1). A more prolonged treatment course for MESCC, typically delivered as 30 Gy in 10 fractions, has the advantage of delivering a higher biologically equivalent dose (BED), which could allow for more durable local control in a disease state where local control directly impacts spinal cord compromise and paralysis (2). However, many patients with MESCC have limited prognoses, and even one week of treatment may impose significant burdens for patients and their caregivers. As a result, shorter courses of palliative radiation therapy in this population can be desirable.

Several previous trials specific to simple, or uncomplicated bone metastases have demonstrated similar efficacy between single-fraction radiotherapy (SFRT) and multi-fraction radiotherapy (MFRT) for symptom relief (3-5). Radiation therapy can provide rapid responses for patients with uncomplicated bone metastases (6), and metaanalyses inclusive of over two dozen randomized trials from McMaster University, the Cochrane review, University of Toronto, and other international investigators have all concluded there is no significant difference in treatment response between SFRT and MFRT (7-11).

Despite increasing data from phase III randomized clinical trials suggesting non-inferiority of SFRT with similar efficacy in terms of pain control and prevention of future fractures or spinal cord compression, as well as ASTRO publishing guidelines (12) advocating for the utilization of SFRT, the usage rates of SFRT remain low. This indicates a general hesitancy for clinicians to adopt SFRT. In fact, an international survey of radiation oncologists published in 2015 inquiring about the use of fractionation regimens for metastatic spinal cord compression found that SFRT was rarely employed at a rate of $8 \%$ to $18 \%$ (13). While existing data to date demonstrate no difference in survival or motor outcomes with five fraction versus ten or more fraction schedules, $73 \%$ of respondents in the United States treat to 30 Gy in 10 fractions in these patients. In Canada and Australia/New Zealand, 20 Gy in 5 fractions is the most commonly used fractionation regimen.

Given this current landscape of treatment patterns for simple bone metastases, the utilization of SFRT for MESCC will certainly remain limited unless more robust data from well conducted phase III clinical trials provide further evidence and reassurance. The previously mentioned bone metastases trials that compared palliative radiotherapy fractionation schedules specifically omitted patients with complex bone metastases and spinal cord compression. The standard of care for patients with MESCC involves 
decompressive surgery followed by adjuvant radiation therapy, based on a seminal study by Patchell et al. (14), which found a greater improvement in patient survival and functional outcomes compared with radiation therapy alone. However, it is estimated that approximately $85-90 \%$ of patients present with widely metastatic uncontrolled systemic disease, multiple medical comorbidities, limited ambulatory status, and multi-level vertebral metastases, all of which make them a poor surgical candidate; these patients have typically been treated non-operatively with MFRT for symptom management that also provides effective pain relief and preservation of neurologic function $(1,15)$.

Until now, three prospective phase III randomized clinical trials compared the effectiveness of SFRT versus MFRT in the treatment of MESCC (16-18), which demonstrated similarity in functional outcome. In Egypt, Abu-Hegazy et al. (19) compared radiotherapy with 8 Gy in a single fraction, 30 Gy in 10 fractions, and 40 Gy in 20 fractions in 285 patients with metastatic spinal cord compression. Although they found no statistically significant difference between the 3 groups with regards to functional outcomes and toxicities, SFRT did lead to a higher rate of in-field recurrences $(\mathrm{P}=0.01)$. An Italian trial by Maranzano et al. (16) examined 8 Gy in a single fraction versus 16 Gy in 2 fractions in 372 patients with MSCC and limited life expectancy. Both groups had a median survival of four months, with no difference in response rates. In addition, Cancer Trials Ireland conducted a non-inferiority trial (ICORG 05-03) comparing $10 \mathrm{~Gy}$ in a single fraction versus $20 \mathrm{~Gy}$ in five fractions in 112 patients with MESCC (17). They found no statistically significant difference in patientreported quality of life or pain scores at five weeks and at three months post treatment.

Although limitations exist for each trial, Donovan et al. (20) recently performed a meta-analysis (that included the trials by Maranzano, ICORG 05-03, and the SCORAD trial discussed below), which was the subject of a recent Annals of Palliative Medicine Editorial Commentary (21). They found no differences in motor response, bladder dysfunction, or survival following radiation treatment with either SFRT or MFRT. However, the authors performed their analyses before the final publication results from two of these three trials.

The largest randomized study to date comparing radiotherapy fractionation regimens for patients with MESCC, The SCORAD trial, was recently published in The Fournal of the American Medical Association by Hoskin et al. (18). This trial evaluated the efficacy of SFRT delivered to 8 Gy versus MFRT delivered to 20 Gy in 5 fractions in patients with malignant metastatic solid tumors and spinal canal compression. The authors enrolled 686 patients in the United Kingdom and Australia with an eligibility criteria of age at least 18 years or greater, an estimated life expectancy of more than 8 weeks, and magnetic resonance imaging or computed tomographic scan demonstrating single- or multisite spinal cord compression. Histologic or cytologic confirmation was required in all cases except for prostate cancer patients with serum prostate specific antigen lever greater than $100 \mathrm{ug} / \mathrm{L}$. Patients who were candidates for chemotherapy or surgery were excluded. They assessed the primary endpoint of achieving ambulatory status of 1 or 2 at 8 weeks with a four-point scale consistent with the World Health Organization performance status, and patient quality of life was measured using the European Organization for Research and Treatment of Cancer (EORTC) Quality of Life Questionnaire-Core Questionnaire (QLQ-C30).

The median overall survival of the cohort was 13.1 weeks, and the median survival time for SFRT and MFRT were 12.4 versus 13.6 weeks, respectively, with no statistically significant difference in survival between the groups [stratified HR, 1.02 (95\% confidence interval (CI), 0.74-1.41); $\mathrm{P}=0.91]$. $69.3 \%$ of patients in the SFRT group reached ambulatory status grade 1 or 2 at 8 weeks vs. $72.7 \%$ in the MFRT group [difference, $-3.5 \%$ (1-sided $95 \%$ CI, $-11.5 \%$ to $\infty)$; $\mathrm{P}$ value for noninferiority $=0.06]$. For all other time points at weeks 1,4 , and 12 , the CI limits were within the noninferiority margin. Overall survival rates at 12 weeks were $50 \%$ in the SFRT vs. $55 \%$ in the MFRT group [stratified hazard ratio, 1.02 (95\% CI, 0.74-1.41)]. Toxicity results were similar between arms (grade 3-4 adverse events $20.6 \%$ with SFRT vs. $20.5 \%$ with MFRT) with the exception of increased bladder symptoms in patients treated to the cauda [34\% SFRT vs. 10\% MFRT; odds ratio (OR), 4.53 (95\% CI, 1.4-15.1); $\mathrm{P}=0.014]$. At 8 weeks, $31.1 \%$ of patients in the SFRT group vs. $20.0 \%$ in the MFRT group experienced abnormal bladder function (unadjusted $\mathrm{P}=0.03$, adjusted $\mathrm{P}=0.08$ ). This differential toxicity finding questions the preferential utility of SFRT in patients with lumbar and sacral MESCC. The authors concluded, however, that SFRT did not meet the criterion for noninferiority for the primary outcome of ambulatory status at 8 weeks, but "the extent to which the lower bound of the CI overlapped with the noninferiority margin should be considered when interpreting the clinical importance of 
this finding".

The strengths of this study include the randomization of a large patient cohort and extensive prespecified secondary endpoints, including assessments of the 8 weeks ambulatory response rate, time to loss of ambulation for the population of patients with ambulatory status of 1 or 2 , 8 weeks recovery of ambulation rate, rates of any additional cancer treatment within 12 months, and rates of postrandomization use of supportive care treatments.

We do, however, recommend caution when extrapolating data from statistically non-significant results despite the primary endpoint overlapping with the lower bound of the CI for the prespecified noninferiority margin. In addition, the diversity of tumor histology was somewhat limited, with favorable histologies of prostate (44\%) and breast cancer $(12 \%)$ making up the majority of the study cohort, as well as no appreciable numbers of radiosensitive histologies (myeloma, lymphoma, or small cell). The composition of tumor type within the study cohort is relevant to the study interpretation since histology has been shown to predict who benefits the most with long- versus short-course radiotherapy $(22,23)$.

While specific inclusion and exclusion criteria were used to find a suitable patient population for the SCORAD trial, the study still had a high attrition from death (approximately $40 \%)$ at 8 weeks. This underscores the difficulty in estimating patient prognosis, as providers often significantly overestimate life expectancy (24). TEACHH model accurately identifies patients with any metastatic cancer receiving palliative $\mathrm{RT}$ at the extremes of a prognostic spectrum ( $<3$ months and $>1$ year) (25). Unfortunately, a similar prognostic model specific to MESCC does not yet exist. In addition to ambulatory status, other factors such as tumor histology, interval time between tumor diagnosis and MESCC, presence of other metastases at the time of radiation therapy, and duration of motor deficits have also been found to be associated with patient survival (2). In general, most radiation oncologists take these prognostic factors into consideration when determining which fractionation schedule to use. However, the high attrition rates from death in the majority of previous trials, including SCORAD, highlights the necessity of additional methods to help clinicians estimate patient survival. Development of a more specific and clinically useful prognostic tool may help clinicians more accurately determine life expectancy and more appropriately select patients for SFRT. Future development of these tools may be aided through the use of machine learning and exert augmentation $(26,27)$.
Another important consideration when interpreting the SCORAD trial is the generally advanced age and limited performance status of the study population. In fact, the median age of enrolled patients was 70 years, including $33 \%$ of all patients 75 years or older. Furthermore, $72 \%$ of patients had an ECOG performance status score of 2 or higher, including 13\% with an ECOG score of 4, defined as completely disabled, unable to carry on any selfcare, and totally confined to a bed or chair. Younger and healthier patients would often be expected to have a longer life expectancy and be suited to tolerate more intense salvage therapies. Undertreating MESCC in these patients may, therefore, put them at increased risk of local recurrence and further spinal cord compression, requiring additional interventions that may not be as effective in the retreatment setting and resulting in a further decline in quality of life.

Despite these caveats, SFRT should certainly play a role in the care of patients with MESCC. Increased emphasis on universal health care and economical expenditures emphasizes the need to optimize resource utilization and improve access to care. SFRT significantly minimizes the duration of medical treatment to those patients with a poor prognosis who are often imminently bound for hospice. Shorter radiation treatment courses would also be particularly relevant for patients in rural areas who more often live greater distances from a radiation oncology center.

The authors of SCORAD should be highly commended for conducting the largest, most informative trial to date on fractionation for MESCC. Due to the patient population and negative primary end point of this trial, we recommend caution when universally endorsing SFRT for all MESCC patients. Subset analyses and examination of patient and disease characteristics from this and prior randomized trials may help further pinpoint the ideal patients to treat with SFRT. Overall, however, our early interpretation of this trial suggests that an elderly patient population with a more limited performance status and single level of disease may be most optimally treated with SFRT, especially when the MESCC is in the cervical or thoracic spine.

\section{Acknowledgments}

Funding: None.

\section{Footnote}

Conflicts of Interest: All authors have completed the 
ICMJE uniform disclosure form (available at http://dx.doi. org/10.21037/apm-20-990). The authors have no conflicts of interest to declare.

Ethical Statement: The authors are accountable for all aspects of the work in ensuring that questions related to the accuracy or integrity of any part of the work are appropriately investigated and resolved.

Open Access Statement: This is an Open Access article distributed in accordance with the Creative Commons Attribution-NonCommercial-NoDerivs 4.0 International License (CC BY-NC-ND 4.0), which permits the noncommercial replication and distribution of the article with the strict proviso that no changes or edits are made and the original work is properly cited (including links to both the formal publication through the relevant DOI and the license). See: https://creativecommons.org/licenses/by-nc-nd/4.0/.

\section{References}

1. Rades D, Šegedin B, Conde-Moreno AJ, et al. Radiotherapy With 4 Gy $\times 5$ Versus 3 Gy $\times 10$ for Metastatic Epidural Spinal Cord Compression: Final Results of the SCORE-2 Trial (ARO 2009/01). J Clin Oncol 2016;34:597-602.

2. Rades D, Panzner A, Rudat V, et al. Dose escalation of radiotherapy for metastatic spinal cord compression (MSCC) in patients with relatively favorable survival prognosis. Strahlenther Onkol 2011;187:729-35.

3. van der Linden YM, Steenland E, van Houwelingen HC, et al. Patients with a favourable prognosis are equally palliated with single and multiple fraction radiotherapy: results on survival in the Dutch Bone Metastasis Study. Radiother Oncol 2006;78:245-53.

4. Sande TA, Ruenes R, Lund JA, et al. Long-term followup of cancer patients receiving radiotherapy for bone metastases: results from a randomised multicentre trial. Radiother Oncol 2009;91:261-6.

5. Kaasa S, Brenne E, Lund JA, et al. Prospective randomised multicenter trial on single fraction radiotherapy $(8 \mathrm{~Gy} \mathrm{x}$ 1) versus multiple fractions (3 Gy x 10) in the treatment of painful bone metastases. Radiother Oncol 2006;79:278-84.

6. Malek L, Chow E, Simone CB 2nd. Speedy response can be achieved from palliative radiotherapy in the treatment of painful uncomplicated bone metastases. Ann Palliat Med 2017;6:S120-1.

7. Wu JS, Wong R, Johnston M, et al. Meta-analysis of dose-fractionation radiotherapy trials for the palliation of painful bone metastases. Int J Radiat Oncol Biol Phys 2003;55:594-605.

8. Sze WM, Shelley M, Held I, et al. Palliation of metastatic bone pain: single fraction versus multifraction radiotherapy - a systematic review of the randomised trials. Cochrane Database Syst Rev 2004;2002:CD004721.

9. Chow E, Harris K, Fan G, et al. Palliative radiotherapy trials for bone metastases: a systematic review. J Clin Oncol 2007;25:1423-36.

10. Chow E, Zeng L, Salvo N, et al. Update on the systematic review of palliative radiotherapy trials for bone metastases. Clin Oncol (R Coll Radiol) 2012;24:112-24.

11. Chow R, Hoskin P, Schild SE, et al. Single vs multiple fraction palliative radiation therapy for bone metastases: Cumulative meta-analysis. Radiother Oncol 2019;141:56-61.

12. Lutz S, Balboni T, Jones J, et al. Palliative radiation therapy for bone metastases: Update of an ASTRO EvidenceBased Guideline. Pract Radiat Oncol 2017;7:4-12.

13. Ryu S, Maranzano E, Schild SE, et al. International survey of the treatment of metastatic spinal cord compression. J Radiosurg SBRT 2015;3:237-45.

14. Patchell RA, Tibbs PA, Regine WF, et al. Direct decompressive surgical resection in the treatment of spinal cord compression caused by metastatic cancer: a randomised trial. Lancet 2005;366:643-8.

15. Rades D, Stalpers LJ, Hulshof MC, et al. Comparison of $1 \times 8$ Gy and $10 \times 3$ Gy for functional outcome in patients with metastatic spinal cord compression. Int J Radiat Oncol Biol Phys 2005;62:514-8.

16. Maranzano E, Trippa F, Casale M, et al. 8Gy singledose radiotherapy is effective in metastatic spinal cord compression: results of a phase III randomized multicentre Italian trial. Radiother Oncol 2009;93:174-9.

17. Lee KA, Dunne M, Small C, et al. (ICORG 05-03): prospective randomized non-inferiority phase III trial comparing two radiation schedules in malignant spinal cord compression (not proceeding with surgical decompression); the quality of life analysis. Acta Oncol 2018;57:965-72.

18. Hoskin PJ, Hopkins K, Misra V, et al. Effect of SingleFraction vs Multifraction Radiotherapy on Ambulatory Status Among Patients With Spinal Canal Compression From Metastatic Cancer: The SCORAD Randomized Clinical Trial. JAMA 2019;322:2084-94.

19. Abu-Hegazy M, Wahba HA. Single-versus multi-fraction radiation treatment for metastatic spinal cord compression: 
functional outcome study. Chin-Ger J Clin Oncol 2011;10:535-40.

20. Donovan EK, Sienna J, Mitera G, et al. Single versus multifraction radiotherapy for spinal cord compression: A systematic review and meta-analysis. Radiother Oncol 2019;134:55-66.

21. Yerramilli D, Simone CB 2nd, Dharmarajan KV. One $\&$ done: treating cord compression with single-fraction radiation therapy. Ann Palliat Med 2019;8:356-9.

22. Rades D, Dunst J, Schild SE. The first score predicting overall survival in patients with metastatic spinal cord compression. Cancer 2008;112:157-61.

23. Jones JA, Simone CB 2nd. Palliative radiotherapy for advanced malignancies in a changing oncologic landscape: guiding principles and practice implementation. Ann

Cite this article as: Han JE, Press RH, Hasan S, Choi JI, Simone CB 2nd. Towards answering the optimal palliative fractionation conundrum: single- versus multi-fraction radiation therapy for spinal cord compression. Ann Palliat Med 2020;9(4):1370-1374. doi: 10.21037/apm-20-990
Palliat Med 2014;3:192-202.

24. Christakis NA, Lamont EB. Extent and determinants of error in doctors' prognoses in terminally ill patients: prospective cohort study. BMJ 2000;320:469-72.

25. Krishnan MS, Epstein-Peterson Z, Chen YH, et al. Predicting life expectancy in patients with metastatic cancer receiving palliative radiotherapy: the TEACHH model. Cancer 2014;120:134-41.

26. Luna JM, Gennatas ED, Ungar LH, et al. Building more accurate decision trees with the additive tree. Proc Natl Acad Sci U S A 2019;116:19887-93.

27. Gennatas ED, Friedman JH, Ungar LH, et al. Expertaugmented machine learning. Proc Natl Acad Sci U S A 2020;117:4571-7. 